\title{
Mechanical, physiological and perceptual responses to different rest interval conditions during consecutive sets of the high-pull exercise
}

\author{
R Sabido a, ${ }^{*}\left(\mathbb{D}\right.$, JL Hernández-Davó b ${ }^{*}$ (iD) \\ a Department of Sport Sciences, Miguel Hernández University, Elche, Spain \\ ${ }^{b}$ Faculty of Health Sciences, Isabel I University, Burgos, Spain. \\ *Corresponding author Email: jlhdez43@gmail.com \\ DOI: https://doi.org/10.34256/ijpefs2111 \\ Received: 12-11-2020, Revised: 06-01-2021; Accepted: 07-01-2021; Published: 07-01-2021
}

\begin{abstract}
Olympic weightlifting movements and their derivates are commonly used within resistance training sessions. The aim of the present study was to assess the influence of different rest intervals (RI) over five sets of the high-pull (HP) on power output performance, lactate concentration [La] and rating of perceived exertion (RPE) responses in trained subjects. Eleven well-trained males attended four testing sessions. The first session consisted of 1 repetition maximum (1RM) assessment. The next three sessions consisted of the same protocol ( 5 sets $\times 6$ repetitions at $80 \% 1 \mathrm{RM}$ in the $\mathrm{HP}$ ) but differing in the RI between sets used $(1,2$ and $3 \mathrm{~min})$. No significant power output decreases over the five sets in any RI condition. [La] did not significantly differ between RI conditions. The 1 min RI condition led to greater RPE values $(6.5 \pm 1.8)$ than both $2(5.4 \pm 1.6)$ and 3 min RI $(5.0 \pm 1.8)$. The present study shows that short RIs (i.e., $1 \mathrm{~min}$ ) can be used by strength coaches to design more time-efficient sessions. The use of RPE during power training sessions should be considered as a sensitive tool to quantify training intensity.
\end{abstract}

Keywords: Olympic weightlifting, Power, Recovery, Rating of perceived exertion, Lactate.

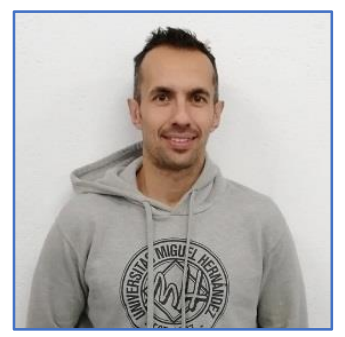

Dr. Rafael Sabido done his Ph.D in Sports sciences and currently he is working as a professor in the in the Department of Sport Sciences, Miguel Hernández University, Elche, Spain.

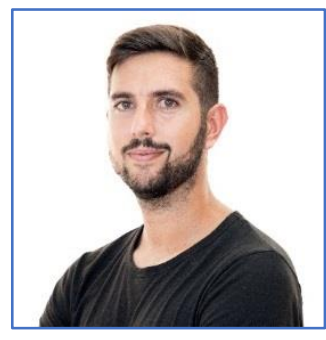

Dr. Jose Luis Hernández Davó completed his Ph.D in sports sciences and currently he is working as a professor in the Faculty of Health Siences, Isabel I University, Burgos, Spain.

\section{Introduction}

Muscular power is an essential variable when aiming to improve sports performance [1]. That is because of the well-established association between the ability to generate maximal power output and performance in jumping, sprinting, and agility tests [24]. Increases in muscular power are a common target for athletes involved in resistance training programs. Among the different ways to improve power performance, the inclusion of Olympic weightlifting (OW) movements and their derivates are commonly used within resistance training sessions [5-7]. A characteristic of these exercises, such as the clean, jerk, or the high-pull, is the necessity of high power to be performed during the execution of an explosive extension of the hip, knee, and ankle joints (commonly referred to as triple extension) [8]. Consequently, they have been proposed as an effective way to optimize power output adaptations, and also to improve 
common sport actions such as jumping and sprinting performance $[9,10]$.

Acute responses and chronic adaptations to resistance training are dependent on the specific selection of training variables (e.g., volume, intensity, density, repetition velocity). Among these variables, the rest interval (RI) between sets has been shown to affect the metabolic, cardiovascular, and hormonal systems [11,12]. Traditionally, research has recommended long RIs during training sessions focused on power development. Power output performance is highly dependent on the phosphagen systems. Thus, Ellington [13] showed that the level of phosphagens present in the muscle fibers is correlated with power output. Long RIs would therefore permit the full replenishment of phosphocreatine stores ( $>4$ min) $[14,15]$, favoring power output maintenance across sets. In contrast, the use of shorter RIs may lead to disturbances in several ion concentrations (e.g., $\mathrm{H}+, \mathrm{Na}+, \mathrm{K}+$ ), resulting in a lowered muscular $\mathrm{pH}$ concentration, determining the athlete's performance in subsequent sets [16]. Previous research has shown that with a lowered intramuscular $\mathrm{pH}$, isometric, rapid force production and power performance are impaired [17-19]. In consequence, the American College of Sports Medicine [20] have suggested the use of long (2-3 min) RIs when performing multi-joint exercises, especially with power aim.

In this line, several studies have shown decreases in power output over multiple sets when using short (i.e., 1 min) RIs in multi-joint exercises such as the bench press [21] and the bench press throw [22,23]. In contrast, Nibali et al., [24] showed no differences in power output decrements when comparing 1, 2, 3 and 4 min RIs across sets in the squat jump exercise using different loads (0-60 kg). Similarly, Martorelli et al., [24] did not find differences in power loss when comparing 1, 2 and 3 min RIs over six sets in the squat exercise. Thus, there seems to be conflicting conclusions about the RI necessary to maintain power output performance during a power training session when using multi-joint exercises. It should be highlighted that the above-mentioned studies differed in the resistance exercises used. Thus, differences in the RI required for upper- and lowerbody exercises should not be discarded.

Despite the very wide use of exercises such as the clean, snatch or the high pull within resistance training programs $[9,25,26]$, little is known about the influence of different RIs between sets on power output responses during a training session. Hardee et al., [27] reported significant alterations in the power clean technique when using short (i.e., 20 s) betweenrepetitions rest. In addition, the use of a cluster sets configuration (20-40 s between-repetition rest) allow for greater power output maintenance compared with traditional (no between-repetitions rest) configuration [28]. Similarly, Haff et al., [29] also showed that the traditional configuration led to peak velocity losses during sets of the clean-pull exercise. Nevertheless, these studies focused on an intraset configuration but not on the RI between sets. Due to the high requirements of power output production, and the great complexity of the OW movements and its derivates, most of the studies including these movements have used long RIs (>3 $\mathrm{min}$ ) between sets $[6,7,30]$. Nevertheless, to the best of the authors' knowledge, there are no studies showing the influence of different RIs on power output maintenance during power training sessions using OW derivates. Therefore, the aim of this study was to investigate the influence of different RI conditions (i.e., 1, 2 and $3 \mathrm{~min}$ ) over five sets of the high-pull exercise on power output performance, lactate concentration [La] and rating of perceived exertion (RPE) responses in trained subjects.

\section{Materials and Methods}

\subsection{Design}

The study followed a within-subject design aiming to evaluate the influence of three different RIs between sets (1, 2 and $3 \mathrm{~min}$ ) on power output performance, [La], and RPE responses after a training session consisting of 5 sets of 6 repetitions of the highpull exercise. Within a 4-week period, subjects attended four testing sessions. The first session was used to evaluate the one repetition maximum (1 RM) in the high-pull exercise. The next three sessions consisted of the same protocol (5 sets $x 6$ repetitions at $80 \% 1 \mathrm{RM}$ in the high-pull exercise) but differing in the RI between sets used. The order of the sessions was counterbalanced among the subjects. All subjects were familiarized with the exercise and all the equipment used. To avoid experimental variability, all subjects were scheduled at the same time for the testing sessions. In addition, the same qualified researcher conducted all testing sessions.

\subsection{Subjects}

Eleven well-trained males (age $=23.8 \pm 5.4$ years, height $=1.79 \pm 0.04 \mathrm{~m}$, body mass $=79.8 \pm$ $8.6 \mathrm{~kg}$ ) voluntarily participated in the study. All subjects had experience in the high-pull movement as 
their habitual training sessions (handball or crossfit) included the use of this exercise. Before participation, all subjects completed a health history questionnaire to document that they were free of any injury and/or illness that may have increased the risk of participation or introduced undesired variability in the study. During the 4-week period of the study, all subjects were instructed to maintain their normal life habits. In addition, subjects were requested to maintain their regular nutritional and hydration state, not to take any nutritional supplementation or anti-inflammatory medications, and to refrain from caffeine intake in the 3 hours before each testing session. Strength training sessions were not allowed at least 72 hours prior to the experimental sessions. Each subject provided written informed consent approved by an Ethics Committee in accordance with the Declaration of Helsinki.

\subsection{High-pull 1RM assessment}

One repetition maximum was performed using a protocol where load was increased incrementally, depending on the previous attempt success. A 3 min RI was used between maximal attempts. The high-pull exercise was performed on a Smith machine (Technogym, Gambettola, Italy). Previous research has shown that power output values in the high-pull exercise did not differ between the Smith machine and the free weight forms $[31,32]$. The exercise began with the barbell starting on the ground. Subjects were instructed to pull the bar in a single, continuous effort through a trunk, hips, knees, and ankles full extension. To be considered a valid attempt, the barbell should exceed the subject's nipple height [5]. In addition to visual inspection, barbell displacement was recorded using a linear position transducer (T-Force, Ergotech, Spain). The maximal weight lifted reaching the aforementioned criterion was considered the individual 1RM. Participant's high-pull 1RM was $76.1 \pm 6.7 \mathrm{~kg}$.

\subsection{Power output testing}

Before testing, all subjects completed a standardized warm-up protocol consisting of 5 minutes' jogging, dynamic stretching, one set of 10 unloaded squats and three sets of eight exercises of the high pull with increasing loads (20, 40 and 60\%). Subjects then performed the experimental protocol consisting of five sets of six repetitions at $80 \% 1 \mathrm{RM}$. The $80 \%$ of $1 \mathrm{RM}$ was used because it is a relative load previously suggested as optimal to maximize power output in the high-pull exercise [5]. The linear position transducer was linked to the barbell, recording data at $1000 \mathrm{~Hz}$.
Then a specialized software application (T-Force Dynamic Measurement System) automatically calculated power data. The values of peak power of each repetition were used for statistical analysis. Subjects were fully encouraged to move the barbell as fast and hard as possible during each repetition.

\subsection{Lactate concentration}

Lactate concentration was determined from capillary blood samples $(0.5 \mu \mathrm{L})$ drawn from the earlobe [33]. After removing the first blood drop, the second drop was collected and analyzed with a portable device (Lactate Scout; Senselab, Leipzig, Germany), with an accuracy of $0.1 \mathrm{mmol}$. Samples were taken 1 minute after each protocol.

\subsection{Rating of perceived exertion}

The OMNI-RES scale (CR-10) was used to determine the subjects' RPE. The scale was defined by the following anchor points: "extremely easy" (0) and "extremely hard" (10). Participants were asked, "How hard do you feel the exercise was?" 5 minutes after the last set of each session.

\subsection{Statistical analysis}

All data were analyzed using the statistical package SPSS 25 (IBM, New York, NY, USA). Data normality was confirmed using the KolmogorovSmirnov test. A single-way repeated measures ANOVA was used to assess differences in [La] and RPE between the different RI conditions. A two-way repeated measures ANOVA ( 3 RI conditions $\times 5$ sets) was used to analyze power output data. When significant interactions were found, a Bonferroni post hoc was used for pairwise comparisons. In addition, the Cohen's d effect size (ES) was used to calculate the magnitude of changes and interpreted as trivial (< $0.25)$, small $(0.25-0.5)$, moderate $(0.5-0.8)$ and large $(>0.80)$. Statistical significance was set at $p<0.05$.

\section{Results and Discussion}

Data of peak power maintenance across sets within each RI condition are shown in Figure 1. There were no differences in inter-set power output performance during the $1 \mathrm{~min} \mathrm{RI}$ and the $3 \mathrm{~min} \mathrm{RI}$ conditions. During the 2 min RI condition, peak power during the 4th set was significantly higher than during the 1st set $(p=0.031)$. Comparisons of peak power in each set between the different RI conditions are shown 
in Figure 2. No significant differences were found in any set $(p=0.476$ to 1.000$)$.

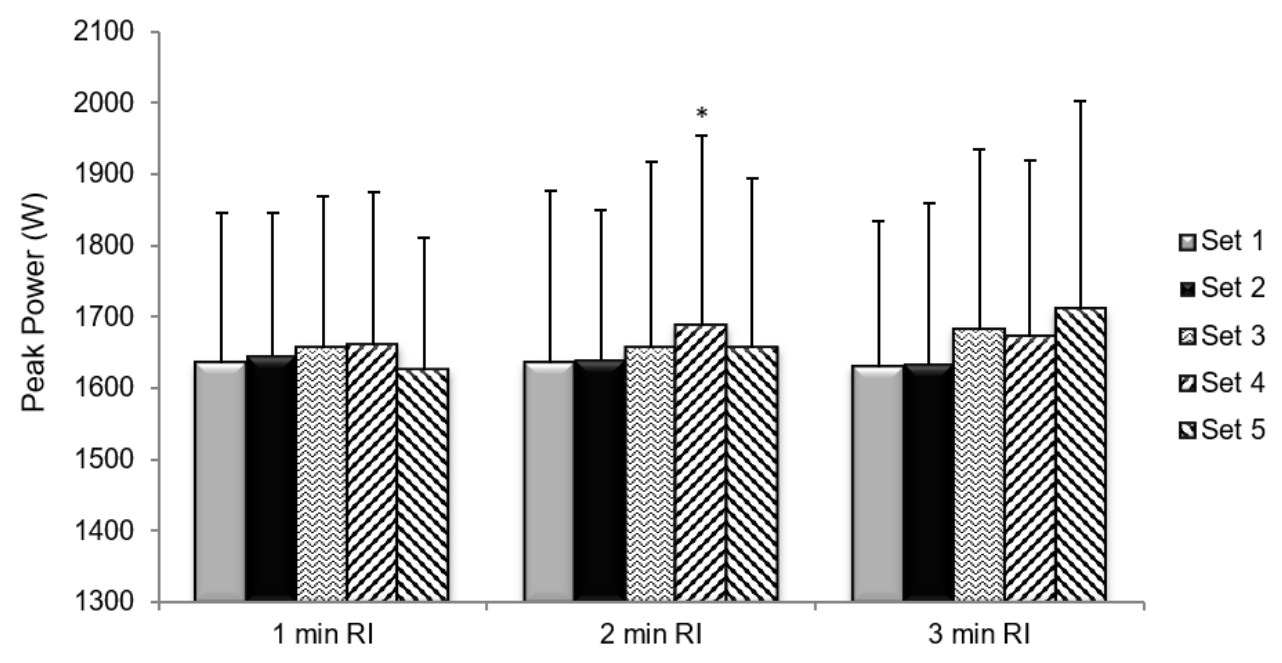

Figure 1. Inter-set peak power comparison within each RI condition. * = significantly higher than the

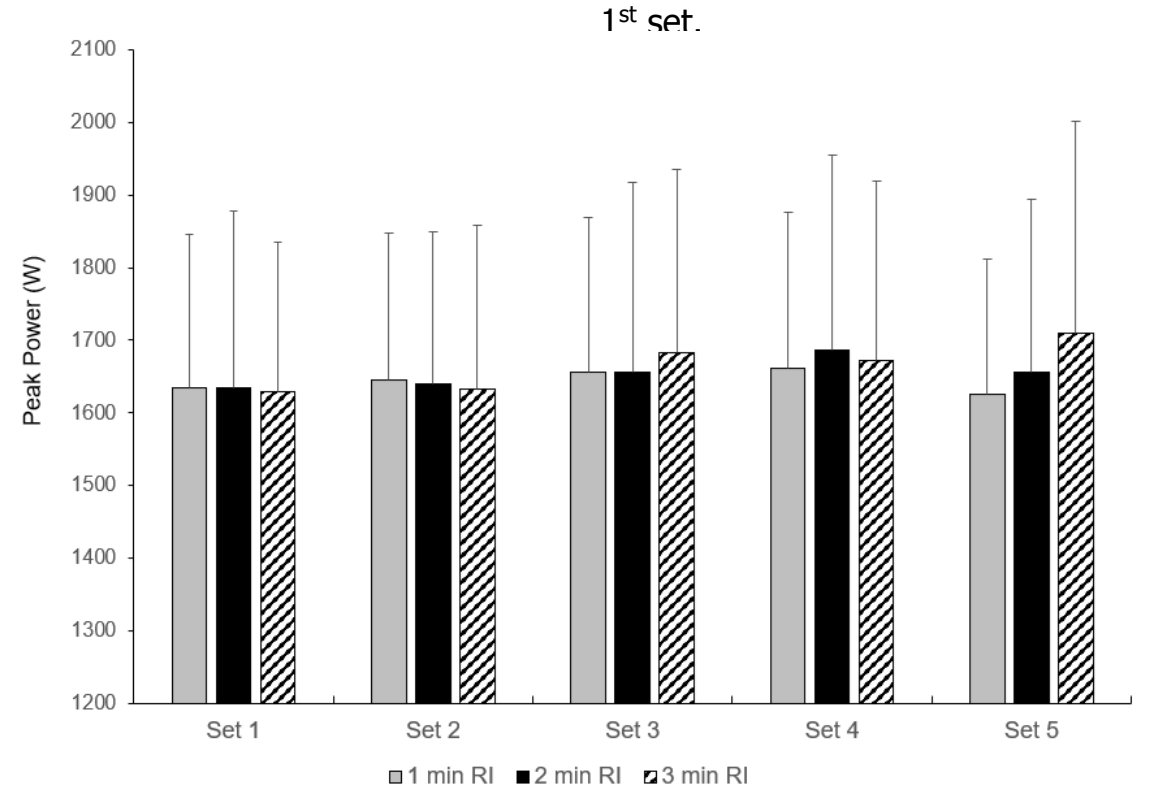

Figure 2. Comparison of peak power between the different RI conditions.

Table 1. Mean percentage change in peak power from the $1^{\text {st }}$ to $6^{\text {th }}$ repetition across sets during each RI condition.

\begin{tabular}{|c|c|c|c|c|c|}
\hline $\mathbf{R I}$ & $\mathbf{1}^{\text {st }} \mathbf{s e t}$ & $\mathbf{2}^{\text {nd }} \mathbf{s e t}$ & $\mathbf{3}^{\text {rd }} \mathbf{s e t}$ & $\mathbf{4}^{\text {th }} \mathbf{s e t}$ & $\mathbf{5}^{\text {th }} \mathbf{s e t}$ \\
\hline $1 \min$ & $-2.95 \%$ & $-3.31 \%$ & $-5.09 \%$ & $-6.15 \%$ & $-4.02 \%$ \\
\hline $2 \min$ & $-2.51 \%$ & $-5.36 \%$ & $-2.75 \%$ & $-3.97 \%$ & $-3.84 \%$ \\
\hline $3 \min$ & $-2.61 \%$ & $-3.20 \%$ & $-1.32 \%$ & $-5.57 \%$ & $-2.09 \%$ \\
\hline
\end{tabular}


Table 2. Displacement and movement duration data across sets for each RI condition.

\begin{tabular}{|c|c|c|c|c|c|}
\hline $\mathbf{R I}$ & $1^{\text {st }}$ set & $2^{\text {nd }}$ set & $3^{\text {rd }}$ set & $4^{\text {th }}$ set & $5^{\text {th }}$ set \\
\hline \multicolumn{6}{|c|}{ Displacement $(\mathrm{cm})$} \\
\hline $1 \min$ & $106 \pm 6$ & $103 \pm 5$ & $104 \pm 6$ & $104 \pm 5$ & $104 \pm 5$ \\
\hline $2 \min$ & $106 \pm 6$ & $105 \pm 6$ & $106 \pm 6$ & $105 \pm 5$ & $105 \pm 7$ \\
\hline $3 \min$ & $106 \pm 7$ & $107 \pm 7$ & $107 \pm 8$ & $107 \pm 7$ & $107 \pm 6$ \\
\hline \multicolumn{6}{|c|}{ Duration (ms) } \\
\hline $1 \mathrm{~min}$ & $851 \pm 42$ & $835 \pm 47$ & $850 \pm 64$ & $856 \pm 67$ & $869 \pm 51$ \\
\hline $2 \min$ & $838 \pm 46$ & $836 \pm 64$ & $837 \pm 62$ & $840 \pm 61$ & $843 \pm 55$ \\
\hline $3 \min$ & $849 \pm 48$ & $854 \pm 58$ & $862 \pm 63$ & $860 \pm 81$ & $856 \pm 78$ \\
\hline
\end{tabular}

Table 3. Comparison of [La] and RPE responses after each RI condition

\begin{tabular}{|c|c|c|}
\hline Rest Interval & [La] & RPE \\
\hline 1 min & $5.4 \pm 0.9$ & $6.5 \pm 1.8$ \\
\hline 2 min & $4.6 \pm 1.3$ & $5.4 \pm 1.6^{*}$ \\
\hline 3 min & $4.9 \pm 1.6$ & $5.0 \pm 1.8^{*}$ \\
\hline * Significantly lower than 1 min RI. & \\
\hline
\end{tabular}

The percentage of peak power change from the 1 st to the $6^{\text {th }}$ repetition of the set during each RI condition is shown in Table 1. The results showed low variations in peak power across sets independently of the RI condition. However, a tendency for slight increases in power output during the $3 \mathrm{~min}$ RI condition can be observed.

Data of barbell displacement and movement duration across sets with the different RI conditions are shown in Table 2. No differences were found in any variable. Results of [La] and RPE after the different RI protocols are shown in Table 3. There were no significant differences in [La] between the RIs, despite a moderate ES value (0.74) when comparing 1 vs. 2 min RI conditions. In contrast, RPE values were significantly higher after the $1 \mathrm{~min}$ RI than after both the $2 \min (p=0.008 ; E S=0.70$, moderate) and the 3 $\min \mathrm{RI}$ condition $(p=0.005 ; \mathrm{ES}=0.86$, large $)$.
The present study was conducted aiming to investigate the influence of different RI conditions ( 1,2 and $3 \mathrm{~min}$ ) across five sets of the high-pull exercise on power output, [La], and RPE responses in trained subjects. The main finding of the present study was that peak power output did not significantly decrease across sets at any RI condition. In addition, [La] did not differ between the different RIs, although RPE values were significantly higher at 1 min RI condition compared with both 2 min and 3 min RI.

The current study showed that either 1,2 , or 3 min RI conditions allowed for peak power output to be maintained across sets. There are conflicting results in the literature regarding the influence of RI lengths on power output maintenance. While some studies have shown significant power impairments when using short (e.g. $1 \mathrm{~min}$ ) RI conditions [21,34], others have suggested a RI of 1 min as sufficient to maintain power performance across sets of the squat [25] and the jump squat exercise [24]. From a physiological point of 
view, power output performance is highly dependent on the phosphagen system. In consequence, some authors have suggested that the inability to maintain power output during high-intensity efforts is mostly mediated by decreases in phosphocreatine (PCr) content [15]. It is most likely possible that the low training volume (six repetitions per set) used in the present study, would not imply the emptying of $\mathrm{PCr}$ stored. Therefore, power output performance may not be impaired. This is in line with Paulo et al. [35] who showed that short RI between sets may fully restore the subject's ability to produce muscle power if low training volumes are used. In addition, previous research has shown that after a high-intensity exercise, an initial fast component of $\mathrm{PCr}$ recovery takes place [36]. Altogether, these factors may explain the nonsignificant decreases in power output found in the present study. Further, results showed no modifications in time or bar displacement across sets, suggesting that power output maintenance is not always linked to changes in movement kinematics [37].

High levels of [La] and the consequent accumulation of $\mathrm{H}+$ ions and $\mathrm{pH}$ decreases have been shown to reduce muscular force and power production [19]. In several studies, the use of a short (1 min) RI between sets caused significant increases in [La] $[21,23]$. In the present study, [La] did not show significant differences between RI conditions. These discrepancies may be explained by differences in the training session characteristics. Abdessemed et al., [21] used a hypertrophy-oriented session, performing sets to failure using a $70 \% 1 \mathrm{RM}$, while in the present study, a protocol consisting of six repetitions with the $80 \% 1 \mathrm{RM}$ load was used. This relative load usually allows for eight repetitions to be performed, which correspond to two repetitions in reserve (RIR). When comparing the responses to a $3 \times 12(\mathrm{RIR}=0)$ with a $3 \times 6($ RIR $=2)$ in the squat exercise, Sánchez-Medina and González-Badillo [38] reported not only greater significant performance losses, but also significant greater levels of ammonia and [La]. Of note is that in the last cited study, the $3 \times 6$ [RIR $=2]$ protocol using the squat exercise showed higher [La] $(7.1 \mathrm{mmol} / \mathrm{L})$ than the values found in the present study (4.6-5.4 $\mathrm{mmol} / \mathrm{L}$ ). However, [La] reported in the current study is similar to studies using Olympic weightlifting exercises [16]. The high-pull exercise involves a high participation of upper- and lower-body and trunk muscles, which implies that the total work performed during the movement is shared among a large number of muscle groups [39, 40]. Although speculative, the utilization of a greater number of muscle groups during the high-pull exercise, may allow for less work to be performed by each muscle group, resulting in lower [La]. Despite trunk and arm muscles participation, the high-pull exercise required a greater participation of leg muscles. Therefore, these low [La] values may be linked to the use of type-I fibers, which predominate leg muscles [41], and are characterized by a higher mitochondrial activity [42].

The RPE scale has commonly been used to monitor individuals' perception of effort during resistance training sessions $[29,43,44]$. In the present study, RPE was the only variable showing differences between the different RI conditions. These results are in line with previous studies where RPE was significantly higher when using short (1 min) vs. long (3 min) RI protocols [23,43]. In a previous study using the high-pull exercise, Hardee et al., [29] showed that decreases in RPE when using long inter-repetition rest were coincidental with decreases in power output. In the current study, RPE but not power output performance showed significant differences between the RI conditions, highlighting the sensitivity of this scale. Therefore, RPE may be considered a valuable tool to monitor perceived effort during power-oriented training sessions.

Among the limitations of the study, we used a sample of young males with experience in resistance training. Considering that variables such as age, sex, or exercise order may influence RI selection, our results cannot be extrapolated to other populations. Although we measure bar displacement, additional kinematic variables (i.e., joint timing and angles) may have provided interesting information. Future studies are required to investigate the chronic effect of these RIs.

\section{Conclusion}

An objective of power training may be to provide sufficient, but not excessive, recovery between sets to guarantee maximal levels of power output at the beginning of the next set. The present study shows that short RIs (i.e., $1 \mathrm{~min}$ ) can be used by strength coaches to design more time-efficient sessions, especially for athletes who employ Olympic weightlifting and its derivates as a support to their discipline and they are time-pressed. The use of RPE during power training sessions should be considered as a sensitive tool to quantify training intensity. 


\section{References}

[1] G.M. Hester, E.C. Conchola, R.M. Thiele, J.M. DeFreitas, Power output during a high-volume power-oriented back squat protocol, Journal of Strength and Conditioning Research, 28(10) (2014) 2801-2805. DOI I PubMed

[2] P. Cormie, M.R. McGuigan, R.U. Newton, Developing maximal neuromuscular power: part 2 - training considerations for improving maximal power production, Sports Medicine, 41 (2011) 125-146. DOI | PubMed

[3] J.M. McBride, N.T. Triplett-McBride, A. Davie, R.U. Newton, The effect of heavy- vs light-load jump squats on the development of strength, power, and speed, Journal of Strength and Conditioning Research, 16 (2002) 75-82. PubMed

[4] L. Suarez-Arrones, O. Gonzalo-Skok, I. Carrasquilla, J. Asián-Clemente, A. Santalla, P. Lara-López, F.J. Nuñez, Relationships between change of direction, sprint, jump, and squat power performance. Sports, 19 (2020) 38. DOI । PubMed

[5] M.J. Barnes, A. Petterson, D.J. Cochrane, Effects of different warm-up modalities on power output during the high pull, Journal of Sports Sciences, 35 (2017) 976-981. DOI I PubMed

[6] A. Chaouachi, R. Hammami, S. Kaabi, K. Chamari, E.J. Drinkwater, D.G. Behm, Olympic weightlifting and plyometric training with children provides similar or greater performance improvements than traditional resistance training, Journal of Strength and Conditioning Research, 28 (2014) 1483-96. DOI I PubMed

[7] W.B. Haug, D.J. Drinkwater, D.W. Chapman, Learning the hang power clean: kinetic, kinematic and technical changes in four weightlifting naive athletes, Journal of Strength and Conditioning Research, 29 (2015) 1766-79. DOI | PubMed

[8] T.J. Suchomel, G.A. Wright, T.W. Kernozek, D.E. Kline, Kinetic comparison of the power development between power clean variations, Journal of Strength and Conditioning Research, 28 (2014) 350-360. DOI I PubMed

[9] F. Arabatzi, E. Kellis, Olympic weightlifting training causes different knee musclecoactivation adaptations compared with traditional weight training, Journal of Strength and Conditioning Research, 26 (2012) 2192-201. DOI I PubMed

[10] W.G. Moore, M.S. Hickey, R.F. Reiser, Comparison of two twelve week off-season combined training programs on entry levels collegiate soccer players' performance, Journal of Strength and Conditioning Research, 19 (2005) 791-8. DOI | PubMed

[11] F.B. de Salles, R. Simao, F. Miranda, J. da Silva, A. Lemos, J. Willardson, Rest interval between sets in strength training, Sports Medicine, 39 (2009) 765-777. DOI I PubMed

[12] H. Mohammadi, M.E. Afzalpour, S.H. Abtahi, Response of creatine kinase and lactate dehydrogenase enzymes to rest interval between sets and set-repetition configuration during bouts of eccentric exercise, Interventional Medicine \& Applied Science, 10 (2018) 83-86. DOI I PubMed

[13] W.R. Ellington, Evolution and physiological roles of phosphagen systems, Annual Review of Physiology, 63 (2001) 289-325. DOI

[14] R.C. Harris, R.H. Edwards, E. Hultman, The time course of phosphorylcreatine resynthesis during recovery of the quadriceps muscle in man, Pflugers Archives, 28 (1976) 137-142. DOI । PubMed

[15] A. Méndez-Villanueva, J. Edge, R. Suriano, P. Hamer, D. Bishop, The recovery of repeatedsprint exercise is associated with $\mathrm{PCr}$ resynthesis, while muscle $\mathrm{pH}$ and EMG amplitude remain depressed, PLoS One, 7 (2012) e51977. DOI I PubMed

[16] A.S. Date, S.R. Simonson, L.B. Ransdell, Y. Gao, Lactate response to different volume patterns of power clean, Journal of Strength and Conditioning Research, 27 (2013) 604-610. DOI I PubMed

[17] D.M. Pincivero, R.M. Campy, The effects of rest interval length and training on quadriceps femoris muscle, part I: Knee extensor torque and muscle fatigue, Journal of Sports Medicine and Physical Fitness, 44 (2004) 111-118. PubMed

[18] J.J. Wang, Z. Qin, P.Y. Wang, Y. Sun, X. Liu, Muscle fatigue: general understanding and treatment, Experimental and Molecular Medicine 49 (2017) e384. DOI I PubMed 
[19] P. Berti, F Donner, C. Guerini de Souza, Effects of beta-alanine supplementation on performance and muscle fatigue in athletes and non-athletes of different sports: a systematic review, Journal of Sports Medicine and Physical Fitness, 57 (2017) 1132-1141. DOI I PubMed

[20] American College of Sports Medicine (ACSM). American College of Sports Medicine position stand. Progression models in resistance training for healthy adults, Medicine and Science in Sports and Exercise 41 (2009) 687-708. DOI । PubMed

[21] D. Abdessemed, P. Duche, C. Hautier, G. Poumarat, M. Bedu, Effect of recovery duration on muscular power and blood lactate during the bench press exercise, International Journal of Sports Medicine, 20 (1999) 368-373. DOI । PubMed

[22] J.L. Hernández Davó, J. Botella, R. Sabido, Influence of strength level on the rest interval required during an upper-body power training session, Journal of Strength and Conditioning Research, 31 (2017) 339-347. DOI I PubMed

[23] J.L. Hernández-Davó, R. Sabido, J.M. Sarabia, J. Fernández-Fernández, M. Moya, Rest interval required for power training with power load in the bench press throw exercise, Journal of Strength and Conditioning Research, 30 (2016) 1265-1274. DOI I PubMed

[24] M.L. Nibali, D.W. Chapman, R.A. Robergs, E.J. Drinkwater, Influence of rest interval duration on muscular power production in the lower-body power profile, Journal of Strength and Conditioning Research 27 (2013) 2723-2729. DOI

[25] A. Martorelli, M. Bottaro, A. Vieira, V. RochaJunior, E. Cadore, J. Prestes, Neuromuscular and blood lactate responses to squat power training with different rest intervals between sets, Journal of Sports Science and Medicine, 14 (2015) 269-275. DOI I PubMed

[26] S.B. Hawkins, T.L. Doyle, M.R. McGuigan, The effect of different training programs on eccentric energy utilization in college-aged males, Journal of Strength and Conditioning Research, 23 (2009) 1996-2002. DOI I PubMed

[27] V. Tricoli, R. Lamas, L. Carnevale, C. Ugrinowitsch, Short-term effects on lower body functional power development: weightlifting vs vertical jump training programs, Journal of Strength and Conditioning Research, 19 (2005) 433-7. DOI I PubMed

[28] J.P. Hardee, M.M. Lawrence, K.A. Zwetsloot, N.T. Triplett, A.C. Utter, J.M. McBride, Effect of cluster set configurations on power clean technique, Journal of Sport Science, 31 (2013) 488-496. DOI | PubMed

[29] J.P. Hardee, M.M Lawrence, A.C. Utter, N.T. Triplett, K.A. Zwetsloot, J.M. McBride, Effect of inter-repetition rest on ratings of perceived exertion during multiple sets of the power clean, European Journal of Applied Physiology, 112 (2012) 3141-3147. DOI I PubMed

[30] G.G. Haff, A. Whitley, L.B. McCoy, H.S. O'Bryant, J.L. Kilgore, E.E. Haff, Effects of different set configurations on barbell velocity and displacement during a clean pull, Journal of Strength and Conditioning Research 17 (2003) 95-103. DOI | PubMed

[31] C. Helland, E. Hole, E. Iversen, M.C. Olsson, O. Seynnes, P.A. Solberg, Training strategies to improve muscle power: is olympic-style weightlifting relevant?, Medicine and Science in Sports and Exercise 49 (2017) 736-745. DOI I PubMed

[32] G.A. Thomas, W.J. Kraemer, B.A. Spiering, J.S. Volek, J.M. Anderson, C.M. Maresh, Maximal power at different percentages of one repetition maximum: influence of resistance and gender, Journal of Strength and Conditioning Research, 21 (2007) 336-342. DOI I PubMed

[33] R.K. Tanner, K.L. Fuller, M.L. Ross, Evaluation of three portable blood lactate analysers: Lactate Pro, lactate Scout and lactate Plus, European Journal of Applied Physiology, 109 (2010) 551559. DOI | PubMed

[34] R. Rahimi, Effect of different rest intervals on the exercise volume completed during squat bouts, Journal of Sports Science and Medicine, 4(4) (2005) 361-366. PubMed

[35] C.A. Paulo, H. Roschel, C. Ugrinowitsch, R. Kobal, V. Tricoli, Influence of different resistance exercise loading schemes on mechanical power output in work to rest ratio -equated and nonequated conditions, Journal of Strength and Conditioning Research 26 (2012) 1308-1312. DOI I PubMed 
[36] S.C. Forbes, A.T. Paganini, J.M. Slade, T.F. Towse, P.A. Meyer, Phosphocreatine recovery kinetics following low- and high-intensity exercise in human triceps surae and rat posterior hindlimb muscles, American Journal of Physiology-Regulatory, Integrative, and Comparative Physiology, 296 (2009) R161-R170. DOI | PubMed

[37] A.O. Natera, M. Cardinale, J.W.L. Keogh, The effect of high volume power training on repeated high-intensity performance and the assessment of repeat power ability: a systematic review, Sports Medicine, 50 (2020) 1317-1339. DOI | PubMed

[38] L. Sánchez-Medina, J.J. González-Badillo, Velocity loss as an indicator of neuromuscular fatigue during resistance training, Medicine and Science in Sports and Exercise, 43 (2011) 17251734. DOI | PubMed

[39] S.K. Chen, M.T. Wu, C.H. Huang, J.H. Wu, L.Y. Guo, W.L. Wu, The analysis of upper limb movement and EMG activation during the snatch under various loading conditions. Journal of Mechanics in Medicine and Biology, 12 (2013) 1350010. DOI

[40] M. Eriksson, M.M. Ekblom, A. Thorstensson, Motor control of the trunk during a modified clean and jerk lift, Scandinavian Journal of Medicine and Science in Sports, 24 (2014) 758763. DOI | PubMed

[41] K. Koppo, J. Bouckaert, A.M. Jones, Oxygen uptake kinetics during high-intensity arm and leg exercise, Research in Physiology and Neurobiology, 133 (2002) 241-250. DOI । PubMed

[42] E. Mygind, Fibre characteristics and enzyme levels of arm and leg muscles in elite crosscountry skiers, Scandinavian Journal of Medicine and Science in Sports, 5 (1995) 76-80. DOI I PubMed

[43] E. Scudese, R. Simao, G. Senna, J.L. Vingren, J.M. Willardson, M. Baffi M, Long rest interval promotes durable testosterone responses in high

intensity bench press, Journal of Strength and Conditioning Research, 30 (2016) 1275-1286. DOI I PubMed

[44] F. Singh, C. Foster, D. Tod, M.R. McGuigan, Monitoring different types of resistance training using session rating of perceived exertion, International Journal of Sports Physiology and Performance 2 (2007) 34-45. DOI I PubMed

\section{Funding \\ No funding was received to carry out this study.}

\section{Ethics Approval}

This study was approved by Institutional ethics committee

\section{Authors Contribution \\ Both authors contributed equally to the manuscript and have read and approved the manuscript (design, data acquisition, analysis, interpretation, manuscript drafting).}

\section{Conflict of interest}

The Authors have no conflicts of interest to declare that they are relevant to the content of this article.

\section{Availability of data and material}

The datasets generated during and/or analysed during the current study are available from the corresponding author on reasonable request

\section{Informed consent}

All participants gave written informed consent to participate in this study.

\section{About The License}

(c) The author(s) 2021. The text of this article is open access and licensed under a Creative Commons Attribution 4.0 International License 